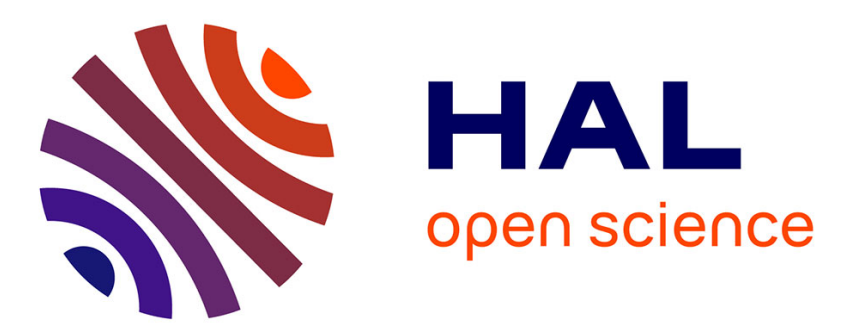

\title{
Le SPD dans le contexte de la crise de la social-démocratie en Europe
}

Etienne Dubslaff

\section{To cite this version:}

Etienne Dubslaff. Le SPD dans le contexte de la crise de la social-démocratie en Europe. Allemagne d'aujourd'hui : revue francaise d'information sur l'Allemagne, 2017, 222, pp.143-148. hal-03185359

\section{HAL Id: hal-03185359 \\ https://hal.science/hal-03185359}

Submitted on 30 Mar 2021

HAL is a multi-disciplinary open access archive for the deposit and dissemination of scientific research documents, whether they are published or not. The documents may come from teaching and research institutions in France or abroad, or from public or private research centers.
L'archive ouverte pluridisciplinaire HAL, est destinée au dépôt et à la diffusion de documents scientifiques de niveau recherche, publiés ou non, émanant des établissements d'enseignement et de recherche français ou étrangers, des laboratoires publics ou privés.

\section{(ㅇ)(1) $\$$}

Distributed under a Creative Commons Attribution - NonCommercial - NoDerivatives $\mid 4.0$ 


\title{
LE SPD DANS LE CONTEXTE DE LA CRISE DE LA SOCIAL-DÉMOCRATIE EN EUROPE
}

\author{
Étienne Dubslaff
}

Association pour la connaissance de l'Allemagne d'aujourd'hui | « Allemagne d'aujourd'hui »

2017/4 N²22 | pages 143 à 148

ISSN 0002-5712

ISBN 9782757420010

Article disponible en ligne à l'adresse :

https://www.cairn.info/revue-allemagne-d-aujourd-hui-2017-4-page-143.htm

Distribution électronique Cairn.info pour Association pour la connaissance de l'Allemagne d'aujourd'hui. (C) Association pour la connaissance de l'Allemagne d'aujourd'hui. Tous droits réservés pour tous pays.

La reproduction ou représentation de cet article, notamment par photocopie, n'est autorisée que dans les limites des conditions générales d'utilisation du site ou, le cas échéant, des conditions générales de la licence souscrite par votre établissement. Toute autre reproduction ou représentation, en tout ou partie, sous quelque forme et de quelque manière que ce soit, est interdite sauf accord préalable et écrit de l'éditeur, en dehors des cas prévus par la législation en vigueur en France. Il est précisé que son stockage dans une base de données est également interdit. 


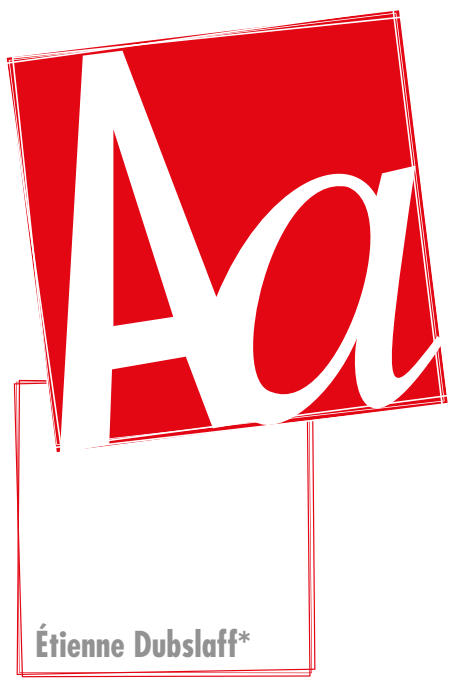

\section{Le SPD dans le contexte de la crise de la social-démocratie en Europe}

\section{Martin Schulz, un homme providentiel ?}

En ce début d'année 2017, les élections législatives fédérales semblent jouées d'avance. Le Parti social-démocrate d'Allemagne (SPD) stagne depuis des mois, si ce $n^{\prime}$ est des années, aux alentours de $20 \%$ des intentions de vote. Il peine en effet à récolter les fruits de son action au sein du gouvernement qu'il forme avec l'Union chrétienne-démocrate (CDU) de la chancelière Angela Merkel et l'Union chrétienne-sociale (CSU) de Horst Seehofer. Pourtant, I'instauration du salaire minimum en janvier 2015, aussi incomplet soit-il, le plafonnement des loyers dans les grandes villes, les quotas féminins dans les conseils d'administration des grandes entreprises sont à mettre au crédit des sociaux-démocrates'. Rien n'y fait, A. Merkel paraît indétrônable. Partant, l'enjeu des élections du 24 septembre semble se résumer à la question de savoir quelles alliances permettraient sa réélection à la tête du gouvernement fédéral.

$C^{\prime}$ est dans ce cadre morose pour la social-démocratie allemande qu'intervient un coup de théâtre peu commun. En ce 24 janvier 2017, alors que tout laisse présager une candidature du ministre de l'Économie et vice-chancelier, Sigmar Gabriel, le président du SPD annonce qu'il s'efface au profit de Martin Schulz. Celui-ci vient de quitter le Parlement européen où il a siégé durant vingt-deux années, dont les cinq dernières en tant que président.

Autant son accession à la scène politique nationale est annoncée, autant il est pressenti comme possible successeur aux Affaires étrangères après l'élection probable de Frank-Walter Steinmeier à la présidence de la République le 12 février 2017. Selon Der Spiegel, Gabriel et Schulz, amis de longue date, étaient convenus dès l'été 2016 qu'ils décideraient d'un commun accord lequel des deux tâcherait de conquérir la Chancellerie. Sans vraiment croire au désistement du premier, Schulz se prépare néanmoins mentalement et physiquement à assumer cette responsabilité. Quoi qu'il en soit, rien ne filtre de leur accord, à tel point que l'intéressé lui-même apprend sa

* Maître de conférences à l'Université Paul-Valéry Montpellier 3, membre du CREG

1. www.fes.de/de/internationale-politikanalyse/aussen-und-sicherheitspolitik/monitor-soziale-demokratie/ strategiedebatten-global/strategiedebatten-deutschland/. Tous les liens hypertextes cités ont été vérifiés le 5 novembre 2017. 
désignation comme candidat et futur président du parti le 21 janvier, trois jours seulement avant la conférence de presse qui le propulse définitivement sur le devant de la scène nationale allemande? ${ }^{2}$.

Cette candidature a de quoi inquiéter le camp de la chancelière. Sur la personnalité, tout d'abord, tout oppose les deux prétendants. Alors que Merkel brille par son sens de la mesure qui confine pour certains à la froideur, Schulz est un passionné. La présidente de la CDU n'aime pas la confrontation ouverte, le social-démocrate en redemande et l'assume à tel point que Die Zeit le met en garde contre lui-même dès début février en posant la question de savoir « combien de Schulz l'Allemagne p[ourrai]t supporter ». S'il a l'avantage de n'avoir alors aucun mandat, I'hebdomadaire conseille à "l'apprenti candidat » d'éviter la surexposition médiatique et de mettre à profit le temps dont il dispose pour élaborer un programme concret ${ }^{3}$. Le même article insiste sur un avantage biographique de Schulz. Sa vie recèle tous les ingrédients d'un bon récit social-démocrate, chose devenue rare à la tête du plus ancien parti allemand depuis les départs de Gerhard Schröder et Franz Müntefering : celui qui a quitté les bancs de l'école sans passer le baccalauréat, a sombré dans l'alcool avant de se ressaisir et de suivre une formation de libraire. Désormais à la tête d'une librairie, il se fait élire maire de la petite ville de province Würselen, dans I'arrièrepays d'Aix-la-Chapelle, à l'âge de 32 ans, puis député européen sept ans plus tard. Ce parcours lui permet de se présenter comme un homme politique chevronné qui connaît et partage les soucis « de ses voisins », des petites gens (" der kleine Mann ») qui « travaillent dur».

Fort de ces atouts, Schulz respire la volonté de conquérir le pouvoir. Et les sondages lui donnent raison : dès début février, son parti est crédité de plus de $30 \%$ des intentions de vote, ce qui le place à deux ou trois points des partis conservateurs, soit dans la marge d'erreur. Il parvient même à égaler le score de ses deux concurrents conservateurs à la mi-mars et à se maintenir dans une marge de deux points jusqu'à fin mars ${ }^{4}$. L'« effet Schulz » autorise d'autant plus les sociaux-démocrates à rêver à une victoire de leur camp que le SPD n'est sorti vainqueur d'une élection que trois fois depuis 1949 : en 1972, 1998 et 2002. L'engovement pour sa personne est tel qu'il est élu président du SPD et candidat à la chancellerie à l'unanimité des voix le 19 mars, chose inouie. Chose non moins insolite depuis 1990, le SPD revendique 10000 nouveaux membres entre février et mars soit un accroissement net depuis $2016^{5}$. La majorité des nouveaux adhérents que mobilise Schulz a moins de 35 ans et est issue des milieux de gauche : il plaît notamment aux fonctionnaires, aux sympathisants des écologistes et aux indécis traditionnellement enclins à voter pour le SPD mais

2. BARTSCH, Matthias / BÖLL, Sven / FELDENKIRCHEN, Markus / HÖHNE, Valerie / KNAUP, Horand / NEUKIRCH, Ralf, "Sehnsucht nach Größe », in: Der Spiegel, n 5/2017, Hambourg, 2017, p. 13-19, p. 13 et FELDENKIRCHEN, Markus, "Mannomannomann », in : Der Spiegel, $n^{\circ}$ 40/2017, Hambourg, 2017, p. 10-27, p. 13.

3. KLASK, Fabian / KRUPA, Matthias, «Wie viel Schulz erträgt das Land ? », in : Die Zeit, $n^{\circ} 6 / 2017$, Hambourg, 2017, www.zeit.de/2017/06/spd-kanzlerkandidat-martin-schulz-wahlkampf-deutschland-tournee.

4. CASPARI, Lisa, "König der Umfragen », in : Die Zeit, 2017, www.zeit.de/politik/deutschland/2017-02/ martin-schulz-spd-cdu-umfragen/komplettansicht et VAILLANT, Jérôme, "L'"effet Schulz" souffle sur le SPD, mais pas encore sur l'Allemagne », in : The Conversation, 2017, www.theconversation.com/l-effet-schulz-sur-le-spdmais-pas-encore-sur-lallemagne-75505.

5. www.n-tv.de/politik/Schulz-beschert-SPD-Mitglieder-Boom-article 19727356.html. 
rebutés par la personnalité de Gabriel ${ }^{6}$. Ces derniers sont particulièrement difficiles à fidéliser, une fois que l'effet du coup de théâtre s'estompe.

Autrement dit, au sortir de l'hiver, Merkel semble en difficulté face à ce challenger qui, du reste, ne fait pas partie de son gouvernement et bénéficie par conséquent d'une plus grande latitude pour critiquer tant sa personne que son bilan. Mieux, tout en étant une personnalité sociale-démocrate de premier plan - Schulz est membre du directoire depuis 1998 -, on ne peut lui imputer la responsabilité directe des réformes sociales du gouvernement Schröder ou l'augmentation de l'âge de départ à la retraite du premier gouvernement Merkel. Il lui est donc plus aisé d'en prôner des aménagements. En outre, il paraît a priori plus difficile de recourir dans ce contexte à la méthode qu'avaient employée les chrétiens-démocrates face à F.-W. Steinmeier ou P. Steinbrück : plus habile à démobiliser les troupes adverses qu'à mobiliser les siennes propres, Merkel s'était refusée à jouer le jeu de la campagne électorale en 2009 et 2013. Et pourtant, une fois encore, elle passe outre les recommandations de ses équipes de campagne et se garde d'attaquer le candidat social-démocrate, au grand dam de celui-ci ${ }^{7}$. De son côté, Schulz suit les conseils de ses collaborateurs et mise sur l'euphorie plutôt que de faire des propositions concrètes ${ }^{8}$.

\section{Les lendemains qui déchantent}

Huit mois jour pour jour après l'annonce triomphale de sa campagne, le candidat doit assumer le pire score du SPD lors d'un scrutin législatif national. Comment expliquer cette déconfiture vertigineuse qui n'a d'égale que son ascension?

Rétrospectivement, le 26 mars 2017 sonne le glas de l'état de grâce. Les élections législatives en Sarre donnent la victoire à la ministre-présidente sortante, la chrétiennedémocrate Annegret Kramp-Karrenbauer. Les perdants ont beau jeu d'insister traditionnellement sur le caractère régional et le manque de représentativité du scrutin - ce Land compte quelque 800000 électeurs -, les résultats des élections régionales rejaillissent toujours sur les responsables nationaux, tant ils sont perçus comme indicateurs de l'opinion publique générale. Après deux mois d'envolée irrésistible, l'élan du candidat social-démocrate s'essouffle. Son entourage ne s'y trompe pas et lui conseille un infléchissement de sa campagne qu'il regretterait par la suite : finie la tournée triomphale des fédérations sociales-démocrates pour ne pas créer de diversion dans les campagnes législatives régionales dans le Schleswig-Holstein le 7 mai et en Rhénanie du Nord-Westphalie, une semaine plus tard. Chose peu commune, le nouveau président du parti cède les feux des projecteurs à Hannelore Kraft, la ministreprésidente sortante de ce qui est, il est vrai, le bastion historique social-démocrate. Autour du week-end pascal, il disparaît complètement de la scène médiatique par égard pour la campagne de la vice-présidente du SPD'. Non seulement il doit accepter que ces campagnes régionales détournent l'attention de la sienne, mais il doit, en tant que président, assumer les trois défaites consécutives d'Anke Rehlinger, Torsten

6. CASPARI, «König der Umfragen », op. cit.

7. FELDENKIRCHEN, "Mannomannomann », op. cit., p. 12 et GEORGET, Jean-Louis, "La démocratie-chrétienne au lendemain de son triomphe : les ambiguités d'une incontestable victoire », in : Allemagne d'aujourd'hui, $n^{\circ}$ 4/2013, Villeneuve-d'Ascq, 2013, p. 88-100, p. 92.

8. BARTSCH / BÖLL / FELDENKIRCHEN / HÖHNE / KNAUP / NEUKIRCH, « Sehnsucht nach Größe », op. cit., p. 13 sq.

9. FELDENKIRCHEN, « Mannomannomann », op. cit., p. 12-14. 
Albig et Hannelore Kraft, qui totalisent respectivement $29,6 \%, 27,3 \%$ et $31,2 \%$ des votes.

Peut-être faut-il déceler dans cette démarche le signe du revers de la médaille de son indépendance. Faute $d^{\prime}$ avoir véritablement mené sa carrière en Allemagne, il manque d'appuis à tous les niveaux du parti et son entourage immédiat est plus rompu aux rouages bruxellois que berlinois. Absent du gouvernement et surtout du groupe parlementaire du SPD, épicentre traditionnel du pouvoir social-démocrate, il manque d'obligés et de relais au sein du parti. Inversement, la « Kampa », le quartier général de la campagne, pourtant habituée aux candidats surprise depuis 2009, ne parvient pas à jouer des avantages de Schulz.

À ces erreurs stratégiques viennent s'ajouter des maladresses de communication qui nuisent à sa campagne. Ainsi, son vieil ami, Gabriel, ne peut s'empêcher de faire des déclarations fracassantes sur la Turquie le 20 juillet alors que le candidat est reçu le jour même par le président français et peut se présenter en homme d'État parfaitement à l'aise sur la scène internationale ${ }^{10}$. Sans s'embarrasser des apparences, I'ancien Chancelier Schröder, que Schulz a fait acclamer à la tribune du congrès de Berlin, devient président du conseil d'administration de l'entreprise pétrolière russe Rosneft, réputée être un sous-marin au service des intérêts politiques et économiques du Kremlin. Le tollé est tel que le président du SPD doit publiquement se distancier de celui qui fait déjà partie des dirigeants de Gazprom depuis qu'il a quitté ses fonctions gouvernementales en $2005^{11}$. Plus anecdotiques mais non moins fâcheuses sont les erreurs de communication commises par son équipe, telle la publication du communiqué de presse revendiquant la victoire du candidat social-démocrate avant même le début du face-à-face télévisé qui l'oppose à Merkel le 3 septembre ${ }^{12}$. Si la plupart des éditorialistes politiques et des sondages donnent la victoire à la chancelière sortante, ils soulignent la piètre prestation des journalistes qui se bornent à des questions sur la politique migratoire, les négociations autour de l'adhésion de la Turquie à I'Union européenne ou les retraites, autant de questions où les deux candidats ont des positions proches. Des sujets plus clivants tels la transition énergétique, l'éducation ou les défis de la numérisation ne sont pas abordés ${ }^{13}$. Aussi Schulz peine-til à montrer ses différences avec la chancelière qui du reste se garde bien de prêter le flanc à une envolée passionnée de la part du candidat social-démocrate, le privant de son principal atout. Le Spiegel révèle que le camp Schulz a soigneusement préparé son poulain au stoïcisme de la chancelière pour éviter qu'il laisse éclater son courroux en plein direct et ne paraisse inutilement agressif en attaquant frontalement son adversaire. D'une manière générale, Merkel tend à exaspérer de plus en plus le président du SPD au cours de la campagne, notamment depuis qu'il sait qu'il ne remportera pas I'élection ${ }^{14}$.

10. Ibid., p. 20

11. www.faz.net/aktuell/wirtschaft/gerhard-schroeder-soll-sogar-aufsichtsrat-chef-bei-rosneft-werden-15223669. html.

12. www.faz.net/aktuell/politik/bundestagswahl/parteien-und-kandidaten/tv-duell-panne-spd-vermeldet-schulzerfolg-zu-frueh-15181468.html.

13. HEIDE, Dana, "Das harmonische Duell », in : Handelsblatt, Düsseldorf, 2017, www.handelsblatt.com/politik/ deutschland/tv-duell-das-harmonische-duell/20277318 et FISCHER, Sebastian, «TV-Duell. Schulz überrascht, Merkel gewinnt », in : Der Spiegel, Hambourg, 2017, www.spiegel.de/politik/deutschland/angela-merkelgegen-martin-schulz-analyse-des-tv-duells-a-1 165944.html.

14. FELDENKIRCHEN, «Mannomannomann », op. cit., p. 22-25. 
Il n'en reste pas moins vrai que le programme du SPD n'est pas très différent de celui de la CDU. Schulz a beau vouloir défendre "les petites gens » et revenir sur " certains abus » des années Schröder desquelles il est moralement coresponsable en sa qualité de membre des instances dirigeantes du SPD. En l'espèce, il propose d'accroître les droits des chômeurs âgés en cours de reconversion professionnelle, d'interdire le recours aux contrats à durée déterminée quand ils ne se justifient pas par l'activité ou encore de revenir à la gratuité de l'éducation du jardin d'enfants à l'université. Ces points s'inscrivent effectivement en faux contre une partie de la politique gouvernementale du SPD des vingt dernières années. Les réajustements que propose Schulz sont cependant marginaux, de sorte qu'ils ne parviennent pas à réconcilier les déçus de la social-démocratie avec le SPD ${ }^{15}$. Inversement, ces concessions peuvent rebuter les électeurs du centre en quête de flexibilisation accrue du monde du travail en vue de renforcer la compétitivité de l'économie allemande.

Les prises de position de Schulz en faveur du mariage homosexuel, de l'égalité salariale entre les hommes et les femmes, de l'intégration européenne renforcée ou celles contre l'augmentation du budget de l'armement ne le différencient guère des écologistes et ne remobilisent pas les milieux sociaux-démocrates traditionnels.

À la faveur de la désindustrialisation et de la numérisation de l'économie, ces milieux sont, du reste, en perte de vitesse en Allemagne et en Europe. De ce point de vue, les $20,5 \%$ du SPD sont à mettre en rapport avec la déconfiture des partis socialistes et sociaux-démocrates européens ces quelques dernières années, que l'on pense aux $6,35 \%$ de Benoît Hamon au premier tour des élections présidentielles de 2017 , aux $22 \%$ récoltés par le PSOE espagnol en 2016 , aux 7,6\% du SLD polonais qui ne compte plus de député national depuis 2015, aux 5,7\% du PvdA hollandais en 2017 ou encore aux 6,28\% du Pasok grec en $2015^{16}$. Nonobstant les spécificités nationales, ces partis sont confrontés aux mêmes difficultés : la désindustrialisation prive les partis de leur milieu originel et les partis socialistes et sociaux-démocrates ne sont pas parvenus à remplacer l'attrait de la lutte des classes par un nouveau combat fédérateur en adéquation avec les enjeux - ou du moins la rhétorique - du XXle siècle. Ensuite, les sirènes de l'équité et de la justice sociale ne séduisent plus les électeurs en proie à la peur du déclassement social. Que ce soit en Autriche, en France ou en Allemagne, les partis sociaux-démocrates ou socialistes ont perdu leur qualité de défenseur des petites gens au profit des populistes d'extrême-droite ${ }^{17}$. Pour ceux qui voient en l'Union européenne la source de tous les maux, Schulz incarne de surcroît le technocrate européen ${ }^{18}$.

15. KLASEN, Oliver, « Was die Kernklientel vom SPD-Wahlkampf hält », in : Süddeutsche Zeitung, Munich, 2017, www.sueddeutsche.de/politik/bundestagswahl-was-die-kernklientel-vom-spd-wahlkampf-haelt-1.3676038.

16. WISDORFF, Flora, "Europas Sozialdemokraten auf dem Weg in die Bedeutungslosigkeit», in : Die Welt, Berlin, 2017, www.welt.de/politik/ausland/article 163963663/Europas-Sozialdemokraten-auf-dem-Weg-indie-Bedeutungslosigkeit.html.

17. RÁSONYI, Peter, « Das Verwelken der Sozialdemokratie », in : NZZ, 2016, www.nzz.ch/meinung/kommentare/leitartikel-das-ende-der-sozialdemokratie-ld.83738, NIESMANN, Andreas / GROSSE HALBUER, Andreas / HALPERT, Marta, "Der Abstieg der SPD und die Frage geht da noch was? ", in : Focus, $n^{\circ}$ 20/2016, Berlin, 2016, www.focus.de/politik/deutschland/in-ganz-europa-darben-die-roten-schaffensich-die-genossen-selbst-ab-drei-gruende-fuer-das-siechtum-der-sozialdemokratie_id_5527387.html et JOFFE, Josef, "Geteiltes Land», in : Die Zeit, n 39/2017, Hambourg, 2017, p. 12.

18. Le camp chrétien-démocrate joue de ces préjugés à l'encontre de Schulz, cf. BARTSCH / BÖLL / FELDENKIRCHEN / HÖHNE / KNAUP / NEUKIRCH « Sehnsucht nach Größe », op. cit., p. 16. 
De son côté, le candidat malheureux voit les raisons de son échec moins dans le positionnement programmatique de son parti que dans le rôle dans lequel il s'est laissé enfermer. Suivant les préceptes de ses conseillers, il a trop tardé à faire des propositions concrètes et s'est résigné à ronger son frein plutôt que d'attaquer frontalement la candidate chrétienne-démocrate. D'autre part, Merkel est parvenue à se présenter comme la chancelière d'une Allemagne qui gagne dans un pays où la grande majorité de la population a le sentiment de bien se porter. Cela condamne le challenger social-démocrate à jouer les ergoteurs pessimistes, un rôle qui se marie fort mal avec sa personnalité enthousiaste et combative ${ }^{19}$. Ce sont ces traits de caractère qui éclatent au grand jour au soir des élections : alors que le score du SPD est historiquement bas, tout comme celui des partis conservateurs du reste, ce n'est pas un Martin Schulz abattu que découvrent les téléspectateurs mais bien un Martin Schulz vindicatif qui entend mener une politique d'opposition énergique. Comment interpréter la nomination d'Andrea Nahles à la tête du groupe parlementaire du SPD ? II s'agit d'une personnalité clivante, expérimentée et classée à l'aile gauche du parti. Schulz a-t-il dû céder la place à une concurrente ou cherche-t-il et à se maintenir au-dessus des débats parlementaires pour mieux se préserver? Quoi qu'il en soit, le positionnement oppositionnel du SPD semble avoir favorisé la victoire inattendue des sociaux-démocrates bas-saxons autour de Stephan Weil lors des élections législatives du 15 octobre. Le SPD y a renoué avec les scores d'un certain Gerhard Schröder en 1998 qui, pour sa part, avait misé sur l'électorat plus centriste de la « Neue Mitte ».

19. MÜHLENDORF, Mira, «Kandidat wird in unpassende Rolle gezwängt. Persönlichkeitsexpertin erklärt : Darum kommt Schulz nicht bei den Wählern an », in : Focus, Berlin, 2017, www.focus.de/politik/experten/kandidat-wird-in-unpassende-rolle-gezwaengt-persoenlichkeitsexpertin-erklaert-darum-kommt-schluz-nicht-bei-waelern-an_id_7627978.html. 